\title{
Dietary fibre and prebiotics in infant formulas
}

\author{
Jacques Ghisolfi \\ Gastro-Enterologie - Nutrition, Hôpital des Enfants, 330 avenue de Grande-Bretagne, \\ BP 3119, 31026 Toulouse Cedex 3, France
}

\begin{abstract}
Prebiotics have the potential to promote immediate and long-term effects on the health and well-being of infants. They have been added to infant formulas in Japan for 20 years and have only recently been used in Europe. The objective is to change the intestinal microflora in order to mimic the bacteriological effect and, thus, the functional effects of human milk. There is, however, a potential risk of long-term effects of the use of these infant formulas. The consequences of using prebiotics in infants, during the first months of life, on the composition and development of the intestinal microflora, and on the resulting bacterial-bacterial and bacterial-host interactions are not known. Attempts have been made to improve infant and follow-on formulas by prebiotic supplementation, but intervention studies are needed to evaluate their immediate and long-term beneficial effects and demonstrate the absence of harmful consequences from their use.
\end{abstract}

\section{Prebiotics: Infant feeding: Dietary fibre}

The main objective of adding prebiotics to infant formulas is to modulate the intestinal microflora, in an attempt to mimic the bacteriological, and consequently, the functional effects of human milk. More generally, the aim is to promote immediate and long-term effects on the health and wellbeing of infants. Prebiotics have been used as ingredients of infant formulas for the last 20 years in Japan, where $90 \%$ of infant formulas now contain prebiotics. In Europe the introduction and use of prebiotics in infant formulas is much more recent, over the last 2 or 3 years.

\section{Prebiotics and infant formulas}

Natural dietary fibre is not used in paediatric nutrition.

Of the large number of prebiotic substances, only four types of prebiotic-supplemented infant formulas have been studied. Their marketing, however, varies from country to country.

Formulas supplemented only with fructo-oligosaccharides (FOS) are not currently used, although there are some reports of infant clinical studies of formulas containing FOS obtained from hydrolysis of inulin.

Infant formulas supplemented with galacto-oligosaccharides (GOS) are marketed in Japan. The GOS is obtained from lactose by an enzymic process. Although GOS have been used as ingredients in infant formulas in Japan for
20 years, there have been few studies of clinical interest in young children.

Human milk oligosaccharides are complex, and it has been postulated that the biological effects of prebiotics in infants could be associated with this mixture of different oligosaccharide structures. Thus, in Europe infant formulas supplemented with a mixture of high-molecular-weight FOS and low-molecular-weight GOS are being marketed and clinical studies in young children using this product are now being published.

Experimental and clinical studies in adult subjects in France have suggested the potential of a new formula containing active metabolites for use in children. These metabolites, which have yet to be isolated, are obtained from the fermentation of milk products with Bifidobacterium breve and Streptococcus thermophilus, and would have the potential to stimulate the growth of bifidobacteria. For this reason they are considered as prebiotics.

\section{Prebiotic-supplemented formulas and intestinal microflora}

The effect on intestinal microflora of any putative ingredient with presumed prebiotic properties should demonstrate the stimulation of bifidobacteria growth, which is the key factor for health benefits. It is well established that the ingestion of 
FOS and GOS leads to an increase in the faecal population of bifidobacteria in animals and in adult subjects. There is at present very little reported data that demonstrates this effect for prebiotic-supplemented formulas.

In adult subjects FOS stimulate the growth of bifidobacteria and possibly lactobacilli, but this action has not been demonstrated in infants. There are only four recently-published double-blind randomised studies that have evaluated the growth of bifidobacteria as a consequence of FOS-supplemented formulas. Two of the studies involved newborn babies and two studies were of children aged from 4 to 10 months who received increasing doses of different FOS over periods varying from 2 weeks to 8 months. The four studies were unable to demonstrate any bifidogenicstimulating effect of the FOS-supplemented diet. This lack of effect may be methodological, representing shortcomings in the bacterial analysis.

Despite intensive use in Japan for many years, there are no published studies in peer-reviewed journals of the effects of GOS-supplemented formulas on bacteria. There have been only two reports of Japanese studies, using the Snow Brand Milk Product, that indicate that a galactosyl-lactose supplement to an infant formula leads to an increase in the faecal counts of bifidobacteria and lactobacilli.

Two studies investigating a mixture of GOS and FOS in infant formulas report a dose-dependent stimulating effect on the growth of bifidobacteria and lactobacilli in the intestine. Moro et al. (2002) analysed faecal samples from sixty newborn infants who had received a formula supplemented with either 4 or $8 \mathrm{~g}$ of a mixture of FOS and GOS/1 for 1 month. Compared with a control group receiving the same non-supplemented formula, a dose-dependent increase in the number of bifidobacteria and lactobacilli in the faecal samples was observed after $28 \mathrm{~d}$. No significant change was observed in other components of the faecal flora, particularly bacteroides, Clostridium spp., Escherichia coli, proteus and klebsiella.

A new French infant formula, which includes metabolites obtained from fermentation of milk products with Bifidobacterium breve and Streptococcus thermophilus, appears to contain active components that, as with classical prebiotics, produce effects on the intestinal microflora. In a randomised double-blind placebo-controlled trial carried out in Lille, France a comparison was made between a supplementedformula group and a non-supplemented-formula group. In this study rectal bacterial flora from thirty-four healthy newborn infants were studied at 1, 2, 3 and 4 months of age (Yazourh et al. 2000). Analyses of bifidobacteria showed an increased count in the supplemented-formula group compared with the non-supplemented-formula group. There was also a decrease in the Clostridium perfringens count. These bacteriological effects have been linked to the presence of components produced during fermentation of the formula that act as prebiotics.

\section{Prebiotic-supplemented formulas and infant health benefits}

Currently, there are few reported studies related to potential health benefits of infant formulas supplemented with prebiotics, and only limited information is available. The results are confined to the FOS- and GOS-supplemented formulas (Moro et al. 2002).

In infants receiving these formulas there was a dosedependent softening of the stools, a decrease in the stool $\mathrm{pH}$ and an increase in the frequency of defecation. During the study period no infant receiving the supplemented formula had diarrhoea.

It would be expected from adult studies that the use of infant formulas supplemented with prebiotics would benefit some aspects of the intestinal immune system. However, very little data is available; only the data from two unpublished studies.

The first study in Jakarta, Indonesia (unpublished results) involved young children, aged 9-11 months, who received $1.7 \mathrm{~g}$ raftilose $(70 \%, \mathrm{w} / \mathrm{w})$ and raftiline $(30 \%, \mathrm{w} / \mathrm{w}) / \mathrm{d}$ over a 10 -week period. During the fourth week of the study the infants were vaccinated against measles. At the end of the study period immunoglobulin $\mathrm{G}$ response to the vaccine was higher in the supplemented group compared with the control group. In the second study (unpublished results) infants aged $7 \mathrm{~d}$ to 4 months randomly received either the new French infant formula containing ingredients obtained from the fermentation of milk products with Bifidobacteria breve and Streptococcus thermophilus, or the non-supplemented control formula. These infants also received an anti-polio injection. Higher anti-polio virus immunoglobulin A levels were found in the group receiving the formula supplemented with the fermentation products compared with the control group.

The findings of numerous experimental and clinical studies involving adult subjects suggest that prebioticsupplemented formulas should have clinically beneficial effects in infants. However, there are no corresponding data for infants. As prebiotics are involved in the modification of stool consistency and frequency and in decreasing faecal $\mathrm{pH}$, their use has been proposed in the treatment of constipation and bowel discomfort. Convincing data for such beneficial effects in infants are needed. In a study in which children aged 4-20 months received a FOS-supplemented formula ( $1 \mathrm{~g}$ oligofructose/d for 6 months) the incidence of diarrhoeaassociated symptoms was reported. FOS consumption was found to be associated with a reduction in febrile events, vomiting, antibiotic use and day-care absenteeism (Saavedra et al. 1999).

A recent random double-blind study in France, involving $>400$ children aged from 6 to 12 months, used over a period of 6 months a formula containing new prebiotic ingredients obtained from the fermentation of milk using Bifidobacterium breve and Streptococcus thermophilus. No significant effect on the incidence of acute diarrhoea was demonstrated, although there was a decrease in the severity of the diarrhoea, which was not significant (Goulet et al. 2001).

Clearly, further studies are necessary to provide conclusive evidence that a particular prebiotic or a mixture of prebiotics is effective in preventing common disease or increasing the well-being of infants.

\section{Safety of prebiotic-supplemented infant formulas}

In adult subjects prebiotic consumption has been studied extensively under normal conditions of use with no adverse 
effects observed. The corresponding data on prebiotic supplemention of infant formulas do not seem to indicate any adverse effects on intestinal transit, $\mathrm{N}$ balance, amino acid metabolism, mineral bioavailability or water balance. A single adult case of anaphylactic reaction to inulin and oligofructose has been reported, but the risk of allergic sensitisation with these formulas is not supported by any observations in children or adults.

The main question concerning the potential risks relates to the long-term effects of the use of these infant formulas on the early and long-term modulation of the complex intestinal microflora. In particular, there is no indication of the consequences of using prebiotics in infants, during the first months of life, on the composition and development of the intestinal microflora, or on the resulting bacterialbacterial and bacterial-host interactions. However, there have been no reports of adverse microbial or immunological effects in experimental or clinical studies involving adults or infants.

\section{Conclusion}

Contemporary attempts to improve infant and follow-on formulas by prebiotic supplementation certainly represent a fascinating concept. Despite recent important advances, the data available concerning these nutritional innovations are too fragmentary to formally establish their immediate and long-term efficacy and safety. There is a need for intervention studies to evaluate their immediate and long-term beneficial effects and demonstrate the absence of harmful consequences from their use.

\section{References}

Goulet O, Thibaut H, Gontier C \& Blareau JP (2001) Less severe diarrheic episodes with consumption of a new fermented infant formula FFC50: a double-blind randomised study in 968 French infants. Annals of Nutrition and Metabolism 45, Suppl. 1, 558Abstr.

Moro G, Minoli I, Mosca M, Fanaro S, Jelineek C, Stahl B \& Boehm G (2002) Dosage-related bifidogenic effects of galacto and fructooligosaccharides in formula-fed term infants. Journal of Pediatric Gastroenterology and Nutrition 34, 291-295.

Saavedra J, Tschernia A, Moore N, Abi-Hanna A, Coletta F, Emenhiser C \& Yolken R (1999) Gastro-intestinal function in infants consuming a weaning food supplemented with oligofructose. Journal of Pediatric Gastroenterology and Nutrition 29, 513Abstr.

Yazourh A, Mullie C, Leroux B \& Romond MB (2000) Reproduction par effet prebiotique de la flore intestinale du nourrisson au sein (Probiotic effect on reproduction of intestinal flora of the infant). Archives Françaises de Pediatrie 17, Suppl. 2, 244s-246s. 